\title{
Journée du 18 novembre au Centre Georges
}

Pompidou

Marie-Thérèse Jones-Davies (éd.)

\section{(2) OpenEdition}

\section{Journals}

\section{Édition électronique}

URL : http://journals.openedition.org/shakespeare/1116

DOI : 10.4000/shakespeare.1116

ISSN : 2271-6424

\section{Éditeur}

Société Française Shakespeare

\section{Édition imprimée}

Date de publication : 1 novembre 1980

Pagination : 14-24

Référence électronique

"Journée du 18 novembre au Centre Georges Pompidou », Actes des congrès de la Société française Shakespeare [En ligne], 1 | 1980, mis en ligne le 01 novembre 2007, consulté le 21 avril 2019. URL : http://journals.openedition.org/shakespeare/1116; DOI : 10.4000/shakespeare.1116 


\section{SOCIÉTÉ FRANÇAISE SHAKESPEARE}

\section{ACTES DU CONGRĖS 1979}

DiRecteur DE LA PUblication

M.T. Jones - Davies 
de Macbeth, Macbeth, lui, devient « une petite fille »(III.iv.106). La même chose se produit dans Le Roi Lear, où la folie peut exprimer le passage d'un sexe à l'autre. Lear souffre de l'hysterica passio, qui est une maladie de la femme. Ce symbolisme n'est pas très éloigné de ce qui apparait aussi dans Antoine et Cléopâtre. Enfin la femme violente, comme Regan, Goneril, n'est pas sans évoquer la Méd ée de Sénèque.

On pourrait distinguer beaucoup d'autres aspects de la femme et de son rôle dans Shakespeare, où elle apparaît tentatrice ou prophétesse, séductrice ou protectrice, dans toute son infinie variété.

Après cet exposé une scène de La Tempête fut présentée par Patrick Baty avec J.P. Belfort, Christine Catala et Hélène Hamon. Elle fut suivie d'un débat sur Comme il vous plaira, animé par Mme Jones-Davies, et auquel participèrent Simone Benmussa, Beno Besson, et Coline Serreau, interprête de Rosalinde dans la mise en scène de celui-ci.

\section{Le 18 Novembre}

\section{L'Homme nu \\ Les Fous et la folie dans Shakespeare}

Ce thème fut présenté par Jean Fuzier:

Nous distinguerons d'emblée, comme le faisaient Shakespeare et des contemporains, et comme nous y invite le programme de cette journée, qui associe Comme il vous plaira et Le Roi Lear, entre le "fool 》 et le «madman 》, le simple d'esprit (parfois aussi bouffon) et le dément authentique. L'un et l'autre avait d'ailleurs sa place à l'asile, mais l'un et l'autre participait aussi d'une sagesse supérieure, à la mesure de ce qui était, aux yeux des hommes raisonnables, sa folie. Fou de Comme il vous plaira,Touchstone-pierre de touche de quoi, sinon du bon sens ? - folie à trois de Lear, recréant, avec son authentique dément (le roi), son simple d'esprit promu au rang de fou professionnel (le bouffon), et son simulateur cherchant sous le masque de la folie une protection immédiate autant que les moyens d'une vengeance ultérieure (Edgar), la structure d'un asile de theâtre mis à la mode quelques mois plus tôt par The Honest Whore de Dekker et Northward Ho ! de Dekker et Webster, sous la double influence, satirico-morale et socio-historique, des " hôpitaux 》 sy mboliques (Wireker, Lydgate, Brant, Erasme, Balsac, Copland) et de l'observation réaliste de l'hôpital des fous, concrétisée par L'Hospidale de' pazzi incurabili de Garzoni (1586), véritable détonateur européen de l'asile au théâtre, qu'il s'agît de l'Espagne, de l'Angleterre ou de la France, ce sont là les deux aspects extrêmes du problème : du plus simple et du plus immédiat, encore qu'il englobe toute la tradition élisabéthaine du bouffon (dont celui de Lear sera l'ultime et le plus grand), au plus riche et au plus complexe, puisque c'est toute une tradition internationale, issue du Moyen Age et modelée d'un pays à l'autre par l'observation de la condition spécifique des aliénés, qui s'exprime finalement dans Le Roi Lear. 


\section{Comme il vous plaira}

On entendit ensuite le témoignage de Mario Gonzalès sur son interprétation du rôle de Pierre-de-Touche dans la mise en scène de Comme il vous plaira par Beno Besson. Jean Fuzier fait remarquer à son propos : «Eriger Touchstone en véritable aliéné, (confondant ainsi les traditions du « fool 》 et du « madman 》) comme le fit Mario Gonzalès était une intéressante remise en cause des données socio-histofiques sur lesquelles on se fonde habituellement pour camper le rôle. La discussion à laquelle il participa, pour intéressante qu'elle fût, ne sembla! guère modifier les convictions traditionnelles». Peut-être faudrait-il ajouter que Gonzalès, qui avait su établir avec son auditoire de Beaubourg un rapport de séduction et d'humour, nous a rappelé par ses propos que les démarches de la recherche historique et celles de la création sont parfois bien différentes. Touchstone appartient incontestablement à la première catégorie, celle des «fools », mais ne faut-il pas accepter comme fait théâtral et comme objet de réflexion que ce comédien ait eu besoin de se référer au Quichotte pour composer son personnage - peutêtre à cause de certains traits communs, bien qu'à des degrés différents; et le gentilhomme de la Manche n'est-il pas aussi, à sa manière, une pierre de touche ?

\section{Le Roi Lear}

La séance suivante fut consacrée à la projection du film vidéo réalisé par la R.A.I. : Le Roi Lear dans la mise en scène de Giorgio Strehler. Ce spectacle fut présenté et commenté par Stephan De Lannoy :

La limpidité de ce spectacle naît de la complexité la plus grande. Le montage des scènes, le jeu, le décor, les costumes et les lumières relèvent, les uns et les autres, de plusieurs styles, de plusieurs domaines métaphoriques : mais leur conjugaison renforce l'efficacité de l'action théâtrale. Les quelques écarts par rapport au texte, moins importants que d'habitude, ont eu pour but cette efficacité.

La première scène est ici la cérémonie de partage du royaume. Elle a le caractère d'un prologue dont les contradictions se répercutent sur toute la représentation. Eclairée de lueurs multicolores, vue à travers le voile qui la masque et qui est la carte d'Angleterre, cette cérémonie semble une projection de lanterne magique, un tableau d'imagerie populaire.

La plus évidente des métaphores du spectacle est celle du cirque. Le décor permanent en est la paroi interne d'une tente de cirque. Cette toile unie, formant cyclorama, n'offre pas de repères spatiaux et temporels. Ces repères procèdent du jeu et du montage à vue des meubles. Les seuls meubles sont un tréteau de bateleur et ses avatars. Ils sont montés à vue par les serviteurs des grands seigneurs - les techniciens du cirque, du théâtre - sur le sol de la scène. Celui-ci est fait d'une couche épaisse de débris de plastique noir, figurant une terre meuble : terre primordiale où les morts sont enfouis et dont les vivants renaissent, familière aux serviteurs qui y travaillent, aux bannis chassés sur la lande, à Cordélia qui semble y engendrer Lear « redevenu enfant ». Les puissants de ce monde ne foulent normalement que les tréteaux. $\mathrm{La}$ base idéologique du spectacle est le travail (théâtral) des serviteurs qui le rendent possible. La clownerie n'est pas immédiatement évidente. La colle- 
rette du manteau du roi n'évoque celle d'un clown que lorsqu'il est en compagnie du fou. Gloster porte un manteau semblable; mais c'est seulement après son supplice qu'il fait voir le visage d'un clown funèbre - dérision plus cruelle qu'une souffrance expressionniste. Cornouailles et Regan ont alors des attitudes de fauves; leur animalité sadique rompt la pudeur de la nature humaine, comme l'ont rompue les rodomontades d'Edmond, que son excitation faisait bondir comme un fauve.

Les puissants de ce monde sont pris sous le feu des projecteurs de poursuite : ce privilège factice des vedettes de music-hall est le signe de leur caducité.

Les errants marqués par la folie apprennent la démarche naturelle de l'homme sur la terre. La tempête autour d'eux n'est sonore et obscure qu'au moment où la chaîne qu'ils forment sort ou entre en scène; sitôt entrée, elle se rompt : une lumière intense les saisit et une musique ténue évoque l'antique grandeur royale. La rupture entre l'obscurité et la lumière prend le public dans l'hallucination de cette folie, comme déjà la rupture de la carte par le roi ou celle du manteau du roi errant, bouleversant la logique des choses. Le jeu des métaphores est soumis à la réalité du rapport aux spectateurs et à l'ídéologie.

$\mathrm{La}$ cérémonie de partage présentait une division nette entre les vieux Lear, Gloster, Kent - et les jeunes. Le spectacle se résout sur l'avant-scène, éclairée par les lumières de la salle, quand Edgar accepte le pouvoir comme une tâche, par une adresse au public dite d'un ton naturel, avec une jeunesse assagie, un «nous » qui inclut les spectateurs.

\section{Table ronde sur la Tragédie}

Lear nous conduisait au cœur de l'univers tragique et servit d'introduction à cette table ronde. On peut regretter qu'elle aît été limitée à des exposés successifs et que le temps ait manqué pour établir un dialogue entre les participants et entre eux et l'auditoire.

J. Jacquot. Entrée en matière :

Il a été convenu de limiter la discussion aux tragédies grecques et élisabéthaines, envisagées dans leur contexte historique et par rapport à notre temps. Même ainsi restreint le sujet est immense. On pourrait partir d'une définition minimum, quitte à nuancer et approfondir en chemin. Un helléniste à qui la dramaturgie élisabéthaine est très familière, H.D.F. Kitto, a proposé celle-ci : " La tragédie est une action dramatique qui s'attaque à quelques-uns des problèmes fondamentaux de l'existence et de la condition humaine ». Cette définition suppose que si l'homme change avec les siècles, il y a cependant des constantes dans sa nature. Et aussi que la tragédie ne propose pas des solutions mais pose des questions, instaure un débat. La condition humaine à laquelle se réfère Kitto implique évidemment l'existence de la souffrance et la nécessité de la mort. L'homme est placé dans une situation qui lui est imposée, sa vie s'inscrit dans le temps cosmique et historique. Il est soumis à une contrainte, et pourtant une marge de liberté lui est laissée. Quand aux problèmes propres à la tragédie et qui concernent les concepts de transcendance, de destin, de liberté, de responsabilité, de justice, ils sont posés différemment selon les croyances religieuses, ou les idées philosophiques du milieu 
historique où telle tragédie a vu le jour. Et bien entendu les hommes d'aujourd'hui font intervenir leurs idées et leurs croyances dans les jugements qu'ils portent sur les œuvres tragiques de jadis.

On pourrait encore citer, à ce propos la définition d'André Bonnard, ou plutôt sa profession de foi humaniste, aboutissement d'une lecture profonde des tragiques grecs. La tragédie, dit-il, est plaisir, elle procure la joie de connaître et de répondre par la connaissance à la douleur. Et cette connaissance relève non seulement de l'intelligence, mais de l'expérience affective et de la volonté. Elle est active et génératrice de valeurs, et nous amène à la recréation de nous-même et du monde.

On ne s'étonne pas que les héros de prédilection de Bonnard soient Prométhée et Antigone. Ce qui paraît important dans sa conception c'est que, malgré l'issue souvent malheureuse, la tragédie n'est pas une école de pessimisme et de résignation. Il réinterprête à sa manière la notion grecque de "catharsis 》 qui assimile la fonction tragique à la purification sacrificielle ou thérapeutique. La tragédie n'est pas une chose subie. Il ne suffit pas qu'elle nous aide à retrouver notre équilibre intérieur. Elle doit susciter une prise de conscience et une attitude active.

"La tragédie est plaisir 》..Cependant la tragédie a été refusée au nom d'un certain hédonisme théâtral qui réclame une fin heureuse et un dénouement conforme à la justice. On trouvera une illustration de cette attitude dans les versions "améliorées 》 du Roi Lear où le souverain déchu retrouve son royaume et où Cordelia épouse Edgar, ou bien dans les commentaires de Samuel Johnson qui avait l'âme si sensible qu'il ne pouvait supporter l'injustice de la mort de Cordelia. On peut parler dans le Lear de Shakespeare d'un silence, d'une absence de la divinité. Les justes y sont exposés sans protection aux pires dangers, et les souffrances de Lear ou de Gloucester sont disproportionnées à leur faute. Mais d'autre part les transgresseurs de la loi morale y subissent le contrecoup de leur transgression et s'entredétruisent. Autrement dit le monde n'est pas entièrement absurde. Ceci est rappelé non pas tant pour rouvrir le débat sur Lear que pour suggérer le type d'interrogation qui surgit de la confrontation avec une œuvre tragique.

Aspects de la tragédie grecque. D'après l'intervention de Jean-Pierre Vernant et quelques-uns de ses textes :

Née au VIe siècle en Grèce, la tragédie connaît au Ve siècle, à Athènes, une brusque éclosion et un déclin rapide. C'est une invention neuve et complète - c'est un moment historique limité et particulier. La tragédie innove radicalement dans le domaine des institutions sociales, des formes d'art, de l'expérience humaine. Les œuvres tragiques expriment et élaborent une vision tragique, une façon nouvelle pour l'homme de se comprendre, de se situer dans ses rapports avec le monde, les dieux, les autres, avec soi-même aussi et ses propres actes. Il n'y a pas de vision tragique en dehors de la tragédie et du genre littéraire dont elle fonde la tradition.

La cité grecque se fait théâtre en instaurant chaque année, pour les fêtes de Dionysos, une épreuve où s'affrontent trois poètes tragiques. La cité se met en scène dans un spectacle ouvert à tous. 
Le jeu tragique - qui emprunte à l'épopée et à la poésie lyrique - se déroule sur deux plans : les protagonistes (personnages individualisés) et le chœur (qui traduit quelque peu la sagesse populaire). Les personnages héroïques deviennent l'objet d'un débat et le héros cesse de se présenter en modèle, il est mis en question devant le public. Et cette interrogation atteint le citoyen de l'Athènes démocratique. Dans la perspective tragique, 1'homme et l'action humaine se profilent, non comme des réalités qu'on pourrait cerner et établir - des essences - mais des problèmes sans réponse, des énigmes dont les double-sens restent toujours à déchiffre.

Une opposition forte et douloureuse se dessine entre les anciennes légendes (le passé de la Cité) et des formes institutionnelles nouvelles. Le conflit entre ce passé et les pensées nouvelles qui s'affirment dans le droit et les institutions creuse une distance au cour de l'expérience sociale : le mythe est vu par l'ceil du citoyen - donc, mis en doute - mais la cité, jugée à partir du mythe, est mise en question ou contestée.

Il y a une tension, donc, entre le passé du mythe et le présent de la polis. Cette tension est perceptible à tous les niveaux de la tragédie : la langue est ambigüe ou équivoque; les mots prennent des sens multiples, les phrases sont comprises différemment par le personnage qui les dit, par le chœur, par les dieux, par le public.

Le droit n'est pas unifié, mais divisé et contradictoire, diverses justices s'opposent, des dieux en combattent d'autres. Le héros est déroutant, incompréhensible : voyant, il est aveugle; innocent, il est coupable; agissant, il est agi. La question que la tragédie conduit à se poser à propos de l'homme à travers ses renversements ou péripéties est la suivante : où est l'origine des actes humains ? En lui ? Hors de lui ? Donne-t-il aux actes leur sens ? Ou bien ses actes, revenant le frapper, ne lui donnent-ils pas la véritable connaissance de ce qu'il est et de ce qu'il a fait sans le savoir et sans l'avoir voulu ? Oedipe résout l'énigme du Sphynx : mais il est, pour lui-même, une indéchiffrable énigme.

La tragédie a également joué un rôle décisif dans la prise de conscience du «fictif »: elle a permis à l'homme grec de s'appréhender lui-même, dans son activité de poète, comme un pur imitateur, comme le créateur d'un monde de simulacres et de fables, constituant, à côté du monde réel, celui de la fiction. De même que dans la traditión de l'épopée le poète n'imite pas la réalité mais la dévoile, la tragédie fait parler et agir devant les spectateurs des iires légendaires. Platon et Aristote tenteront d'établir le statut, la place et la fonction de ce qui, pour nous, est devenu « l'art 》: ce sera la théorie de la mimesis - de l'imitation. La parole du poète ne représente pas, elle rend l'être présent. Et les figures de l'âge héroïque que la tragédie met sous les yeux du public - figures qui, pour les Grecs, ne sont pas celles d'êtres fictifs elle leur permet d'être là réellement en même temps que de ne pas y être, car ils relèvent d'un véritable au-delà. Au théâtre, le public n'a pas devant les yeux des hommes dont l'absence est comme impliquée par la narration : mais des personnages subissant des épreuves se produisant devant lui, sous ses yeux, en revêtant les formes de l'existence réelle dans l'actualité du spectacle.

C'est cet aspect direct du discours et de l'action qui constitue, selon Platon, le propre de la mimesis. Miméisthai - imiter - c'est simuler la présence effective d'un absent. Alors, la conscience de la fiction - grâce à la conscience 
du simulacre - est constitutive du spectacle dramatique : elle apparaît à la fois comme sa condition et comme son produit.

On comprend pourquoi les grands événements du temps, les drames de la vie collective n'apparaissent pas comme de ceux qu'on peut transposer sur la scène du théâtre. Le poète Phrynicos avait, dans La prise de Milet, porté sur la scène en 494 le désastre que deux ans auparavant les Perses avaient infligé à la cité de Milet : les spectateurs fondirent en larmes et il fut puni de mille drachmes d'amende. L'histoire - par opposition à la légende - ne permet pas cette distanciation grâce à laquelle les sentiments de terreur et de pitié sont déplacés dans un autre registre, non plus éprouvés comme dans la vie réelle, mais d'emblée compris dans leur dimension de fiction.

Contrairement à l'histoire, la tragédie ne raconte pas, parmi tous les événements qui auraient pu se produire, ceux qui sont effectivement arrivés : en réorganisant, selon ses propres critères, la matière de la légende, en ordonnant la progression de l'intrigue suivant la logique du probable ou du nécessaire, elle montre comment les événements humains, par une marche rigoureuse, peuvent ou doivent arriver. Aussi constitue-t-elle, dans l'esprit du philosophe qui la compare à l'histoire, une création plus sérieuse et plus philosophique. Grâce à la liberté que lui assure le fiction du múthos, elle atteint le général. Le mode d'existence des événements de la tragédie étant imaginaire, ils sont mis à distance en même temps que représentés. Chez le public, désengagé par rapport à eux, ils «purifient » les sentiments de crainte et de pitié qu'ils produisent dans la vie courante. Et s'ils purifient, c'est qu'ils apportent, grâce à l'organisation dramatique, une intelligibilité que le vécu ne comporte pas.

Le drame antique explore les mécanismes par lesquels un individu, si excellent qu'il soit, est conduit à sa perte, non sous l'empire de la contrainte ni par l'effet de sa perversité ou de ses vices, mais en raison d'une faute, d'une erreur comme celles que chacun peut commettre. Par cette voie elle propose au spectateur une interrogation, à portée générale, sur la condition humaine, ses limites, sa nécessaire finitude.

Nicole Loraux : Quelques propositions sur la tragédie grecque comme mise en scène de la féminité.

La tragédie comme lieu de la tension et de la déchirure (voir l'exposé de J.P. Vernant) : déchirure introduite à l'intérieur d'une institution cardinale comme le mariage par son croisement avec une trame empruntée à un passé mythique (ainsi, dans l'Ion d'Euripide, le mariage légitime athénien et le mariage aristocratique se détruisent l'un l'autre), déchirure introduite à l'intérieur d'un langage par son application à un univers relevant d'une autre langue...

Ainsi la déchirure est le produit d'une interférence généralisée entre des mondes qui jamais ne devraient se croiser, mais qui, dans la pratique institutionnelle, ont leur ordre et leur place.

Dans cette perspective, il n'est pas étonnant que, sur la scène tragique, la cité soit mise en question par la féminité.

Féminité des femmes, à la fois pensée comme le danger même et investie, parfois, de toute la légitimité : Clytemnestre, ou le danger même de la femme 
qui fait l'homme, Créuse (dans l'Ion) ou l'étonnante figure d'une «Athénienne» - le mot, dans la langue, n'existe pas, la chose, sur la scène tragique, est incarnée - : une Athénienne porteuse de légitimité, qui transmet le nom athénien, le pouvoir et la terre d'Athènes (le genre historique n'a pas de place pour les femmes; du moins n'en a-t-il pas pour parler du monde grec et du bon fonctionnement des cités, mais dans le monde barbare ou lors de ce déréglement qu'est la guerre civile apparaissent les femmes : Hérodote donne un grand rôle aux femmes de tyrans ou de dynastes, et Thucydide les met en scène dans les guerres civiles; parce que la tragédie dérègle tous les ordres, les femmes $\mathrm{y}$ ont toujours leur place). Féminité des femmes, problématique dans la cité et qui pousse à les représenter en position d'homme.

Mais - et c'est là l'effet capital de la déchirure tragique - la féminité des femmes y. est aussi pensée dans son rapport douloureux à la « race des femmes ", monde clos sur lui-même et que la cité intègre bien mal, au corps des femmes, traversé de passions et de fulgurances, à l'impossible et pourtant bien réelle sagesse des femmes. Et, ultime retournement tragique, apparaissent sur la scène des hommes féminisés par leur confrontation avec les plus féminines des femmes (ainsi, chez Sophocle, Héraclès tué par l'amour de Déjanire et qui pleure des pleurs de femme).

Façon, pour ce genre civique qu'est la tragédie, de faire glisser la cité sur elle-même, vers le temps du mythe, vers le monde des palais, mais aussi, hic et nunc, vers une réflexion sur la division des sexes, l'une des divisions essentielles qui déchirent la cité.

\section{Aspects de la tragédie élisabéthaine.}

Robert Ellrodt :

La tragédie grecque a un horizon religieux au delà des limites de la vie humaine. "Qui sait si ces lois sont saintes chez les morts », interroge Antigone. Mais cet au-delà est mystérieux. "Qui sait ? », "peutêtre », sont des mots qui reviennent. Point de dogme défini, point de réponse aux interrogations.

La théologie chrétienne prétendit apporter des réponses. On sait l'image humaine offerte par Bède le Vénérable : un moineau chassé par la tempête entre dans une salle où flambe un feu clair, puis retourne au froid et à la nuit. Ainsi la vie humaine est visible pour un temps, mais de ce qui suit ou de ce qui a précédé nous ne savons rien. «Si donc cette nouvelle doctrine offre quelque chose de plus sûr il faut l'épouser », conclut un noble Saxon. La religion chrétienne épousée le tragique disparaît. Au théâtre comme en l'épopée le Moyen Age célèbre la Divine Comédie.

Le sentiment tragique a-t-il attendu pour renaître l'agnosticisme de la tragédie jacobéenne ? Tragédie où le moment de la mort est l'entrée "dans la brume ». L'arrière-plan chrétien certes demeure présent : dans Hamlet, dans Lear, dans Macbeth I'homme est entre le ciel et l'enfer. Mais Cordelia morte dans les bras de Lear est-elle devenue ce qu'elle était apparue déjà dans sa vision : "une âme en paradis»? Nul ne le sait. Le reste est silence pour Hamlet, à qui le fantôme lui-même n'a pas révélé « les secrets de sa prison », au point que le prince de Danemark hésite à la frontière du pays 
inconnu d'où nul ne revient. Macbeth et Lady Macbeth se savent damnés, mais cet enfer est-il à venir ou dans leur âme ? Théâtre chrétien, oui, mais où le frisson nouveau du tragique dissout les certitudes.

Et le drame shakespearien a d'autres mystères. La motivation des actes essentiels nous échappe. Pourquoi Brutus a-t-il décidé la mort de César avant de la justifier? Le revirement de Coriolan s'accomplit dans le silence. "C'est la cause 》 dit Othello devant Desdémone endormie, et l'on dispute encore sur la cause. Hamlet mourant murmure «O I could tell you », mais il s'interrompt: «let it be... ».

Point de tragique sans souffrance illimitée. Le châtiment excède la faute. Point de tragique sans l'alliance mystérieuse de la liberté et de la nécessité.

Le destin tragique n'est ni la roue de la Fortune médiévale, ni l'idée chrétienne de la Providence. Le «Dieu caché » du jansénisme est le seul Dieu chrétien qui soit tragique. Il faut que le destin soit subi, mais choisi, que le héros soit libre et son acte inéluctable. Un poignard, «vision fatale », semble conduire Macbeth vers Duncan endormi, mais il lui montre un chemin qu'il avait déjà pris : "Thou marshall'st me the way that I was going».

D'où l'ironie tragique. Les actes libres des personnages les conduisent aveuglément vers l'événement qui change leur vie en destin. "There's a divinity that shapes our ends... ». Hamlet ne sait agir que spontanement, mais il se sent conduit, sans savoir vers quoi. C'est en mourant que le héros a la révélation de ce qu'il était né pour accomplir. Et c'est en devenant aveugle, comme Lear et Gloucester, qu'il apprend à voir clair.

Je ne prétends pas que les «tragédies » de la Renaissance anglaise répondent toutes à cette exigence. Le tragique souvent s'y noie dans l'horreur, s'égare dans la vengeance, se dévore dans le machiavélisme. Vengeance et machiavélisme suscitent des drames puissants, mais l'essence du tragique est ailleurs.

Les passions de crainte et de pitié en toute tragédie sont «purgées » par la distance esthétique que le vers accentue. Mais la distance n'est possible que si l'imagination n'est pas uniquement sollicitée par l'horreur. Joyeuse ou non "l'orgie de l'horrible » éloigne du tragique. Entendons-nous : je ne défends pas un idéal classique de retenue et de dignité. Il y a du grotesque dans la nudité d'Edgar, la folie de Lear, mais en contrepoint d'une grandeur authentique, stö̈cienne ou naturelle. Au moment même où Lear se dépeint « a poor infirm, weak and despised old man » il est - il doit être - royal : «every inch a king ». Point de tragique dans l'abaissement, la déchéance, l'enlisement. Prométhée, Oedipe, Antigone, "inflexibles courages », ne cèdent pas au malheur. L'émoi tragique naît quand la défaite physique est l'occasion d'un triomphe moral. Quand sur la lande où le ciel déverse l'eau et le feu Lear s'écrie «Pour on, I will endure », il est comme l'homme pascalien, plus noble que l'univers car l'avantage que l'univers a sur lui l'univers, n'en sait rien.

Pour changer de langage, disons que le tragique est le moment où la pulsion de mort et la pulsion de vie s'élèvent ensemble à leur degré extrême d'intensité à travers la splendeur du verbe. Point de tragédie sans ce reflet de splendeur, le reste n'est que drame.

Marie-Thérèse Jones-Davies :

Il me semble que deux schémas ou plutôt deux mythes rendent compte des tragédies de la Renaissance anglaise : la chute d'Icare; le choix de Promé- 
thée, qui montrent la destruction de l'homme qui a osé trop tenter. Toujours donc à l'arrière-plan se profile la roue médiévale de la Fortune. On pense à Boccace, au De Casibus Virorum Illustrium ou à Chaucer, au début du Conte du Moine. Cette roue de la Fortune réapparaît d'une tragédie à l'autre, du Tamerlan ou du Faust de Marlowe au Séjan de Jonson, où l'on suit la carrière de celui qui, le matin, est très haut placé et, avant le soir, git au ras de la terre. Le roi Lear est lui aussi enchaîné à une roue de feu. Dans son Apologie de la Poésie, Sidney écrivait que la tragédie enseigne les incertitudes du monde en montrant sur quels fondements tragiques se construisent les toits dorés.

Le héros de Shakespeare est amené par le choix qu'il fait à s'insérer dans la réalité d'un monde où l'ordre auquel il croyait se trouve affronté à un ordre contraire. L'univers de Iago, d'Edmund, de Lady Macbeth... s'oppose à l'univers de Desdémone ou de Cordelia. Au temps de la Renaissance on continue à représenter en termes moraux de fautes, de péchés les causes de la tragédie; mais ce péché commence à être vu comme porteur d'ambiguïté. La voie est ouverte aux paradoxes, qui consistent à dire que «le beau est laid et le laid est beau ». Plus rien n'est stable: «rien n'est plus que ce qui n'est pas ». On définit de fait l'univers présent par la négation de l'ancien. De facto, la représentation du monde sur la scène par le poète tragique crée la relativité.

La construction même de la tragédie où se déroulent deux ou trois histoires simultanées qui se font écho renforce l'ambiguïté d'un monde où rien n'est sûr, où la seule sagesse peut être celle du fou.

Le chœur a presque toujours disparu chez Shakespeare, mais il est remplacé par les commentaires, les apartés, ou les propos d'hommes simples, comme le portier de Macbeth, l'ami d'Antoine, Enobarbus, ou le discours du fou. Souvent par eux s'introduit le rire et la relativité de toutes choses est encore mise en valeur par la juxtaposition des " cornemuses et des funérailles », selon l'expression de Sidney.

Richard Marienstras :

Certains traits de la tragédie élisabéthaine sont - prémonitoirement - assez bien décrits dans des vers du Conte du Moine de Chaucer que l'on cite souvent (et dans certains autres que l'on ne cite jamais).

Voici quelques-uns de ces vers :

Tragedie is to seyn a certeyn storie,

As olde bookes maken us memorie,

Of hym that stood in greet prosperitee,

And is yfallen out of heigh degree

Into myserie, and endeth wrecchedly.

And they ben versified communely

Of six feet, which men clepen exametron.

In prose eek been endited many oon,

And eek in meetre, in many a sondry wyse.

(2770-2778)

I wol biwaile, in manere of tragedie,

The harm of hem that stoode in heigh degree,

And fillen so that ther nas no remedie

To brynge hem out of hir adversitee. 
For certein, whan that Fortune list to flee, Ther may no man the cours of hire withholde. Lat no man truste on blynd prosperitee; Be war by thise ensamples trewe and olde.

(2788-2795)

(Ed. de A.C. Cawley, révisée en 1975).

Chaucer parle bien sûr de "tragédies 》 narratives à la manière de Boccace. Mais les auteurs élisabéthains vont s'emparer de ces narrations tragiques pour en faire éclore, à la scène, la problématique et les contradictions.

La tragédie, on le voit, c'est d'abord une histoire d'un certain type (certeyn storie) - ou peutêtre une histoire attestée : en tout cas, une narration. Narration exemplaire qui concerne les grands de ce monde -.. du monde réel, légendaire ou sacré - et qui relate leur chute, une chute souvent chantée par les poètes ou narrée par les faiseurs d'histoires : mais vers ou prose, peu importe. L'accent est mis sur la narration, le récit ou le poème -.. quelque chose que l'on raconte et qui se situe par conséquent dans le passé.

Cette histoire est celle d'un déclin ou d'une chute catastrophique, qui fait passer le héros de la grandeur à la plus extrême misère. Cette chute est irrémédiable (ther nas no remedie) : elle se situe dans un lieu d'où il n'y a pas de retour, elle conduit à une situation sans rachat.

Toute narration est une manière de se saisir du temps : mais de façon privilégiée, la tragédie est une thématisation du temps. Elle s'empare de son caractère spécifique sur le plan de l'existence humaine : pour l'homme, le temps est irréversible et limité. Le protagoniste de la tragédie ne meurt pas de mort "naturelle ». La finitude humaine est en soi scandaleuse et inacceptable : une vie qui s'achève avant son terme «naturel » fait éclater le scandale. Les auteurs élisabéthains sauront ainsi, en choisissant le protagoniste - - un être hors du commun - montrer le caractère unique et irremplaçable de la personnalité : un héros qui meurt, c'est comme une espèce qui s'éteint. En ce sens, par rapport au théâtre antérieur - celui des mystères ou des moralités -. le théatre élisabéthain est fondateur de la personne. La lamentation - ou la pitié - (I wol biwail...) est constitutive de l'individu dont la disparition est déplorable

- et cela, parce que le protagoniste porteur d'héroïsme, de valeur ou d'amour, est mû par un élan qui devrait le rendre immortel : or, sa course, bien au contraire, le conduit à la mort. Ce qui fait dire fort justement à Northrop Frye que le mouvement de la tragédie est sacrificiel, car on y voit l'infini prendre la même direction que le fini.

La cause de la chute n'importe guère : elle peut être méritée ou imméritée au regard de la morale dominante - comme dans ces petites narrations tragiques de Chaucer où le protagoniste (disons, Lucifer ou Adam) est tantôt puni pour ses fautes, et tantôt (Hercule, Pierre de Castille) abattu sans raison à cause de l'instabilité de la Fortune. La Fortune, en effet, n'épargne personne : la chute des hommes vertueux signifie que le théâtre où se joue le destin des hommes est avant tout ce monde-ci - non ce monde-ci et quelque monde à venir. La problématique de la Fortune, si riche et si variée chez Shakespeare, inscrit la tragédie dans les cassures de l'idéologie dominante : la « vision du monde élisabéthaine »ne peut donc rien «expliquer » de ce qui est tragique : c'est au contraire la tragédie, en une innovation sans précédents dans le monde 
chrétien, qui donne à voir et à vivre l'effondrement des certitudes. De sorte que la tragédie élisabéthaine, qui constitue l'individu, en même temps le problématise et le déconstruit : «Who is it that can tell me who I am? » demande le roi Lear.

L'opposition entre le «train du monde » et la «vision du monde » conventionnelle joue le même rôle, dans la tragédie élisabéthaine, que dans la tragédie grecque l'opposition entre le présent de la polis et le passé du mythe dont parle Jean-Pierre Vernant. Mais l'histoire, interdite dans le théâtre grec, prend ici la place de la légende - l'interdit ne portant que sur l'histoire contemporaine.

Entre l'histoire mouvante ou problématique et le sacré que l'on voudrait immuable se dessine un conflit au terme duquel le sacré est mis en question. Le protagoniste est alors porté à la limite des mondes connus : à la limite du réel, du symbolique, du spirituel - en un lieu où nature et culture abdiquent :

What if it tempt you toward the flood, my lord,

Or to the dreadful summit of the cliff,

That beetles o'er his base into the sea,

And there assume some other horrible form,

Which might deprive your sovereignty of reason

And draw you into madness?

$$
\text { (Hamlet, I, iv) }
$$

Dans cette métaphore saisissante, la chute possible est à la fois matérielle et mentale. On peut donc dire aussi : la tragédie se situe au delà de l'organisation conceptuelle du monde, elle est à la fois une expérience et un effort pour rendre intelligible ce qui ne l'est pas.

$\mathrm{La}$ 《vie aux confins 》 donne leur stature exceptionnelle aux personnages tragiques de Shakespeare : après leur mort, c'est une vie plus pauvre et plus pâle qui reprend, à un niveau ontologique inférieur. Le roi Lear meurt. Mais la « chute ontologique » est subie par les suivants :

The oldest hath borne most : we that are young

Shall never see so much, nor live so long.

On revient ainsi à Chaucer : la tragédie se situe nécessairement dans le passé. De sorte que - dit encore Frye - notre sentiment devant l'expérience tragique est le suivant : l'hérö̈que et l'infini ont été - l'humain et le fini sont.

\section{A L'ECOLE NORMALE SUPERIEURE}

\section{Rencontres avèc des metteurs en scène}

\section{Le 16 Novembre}

Kings. Commentaires de Denis Llorca sur sa mise en scène. Discussion animée par Marie-Claude Rousseau.

Kings, cette fresque royale inspirée des pièces historiques (principalement les trois Henri VI et Richard III mais avec, en toile de fond, toutes les guerres dynastiques de Richard II à Henri VII) est un spectacle " colossal », une fête 